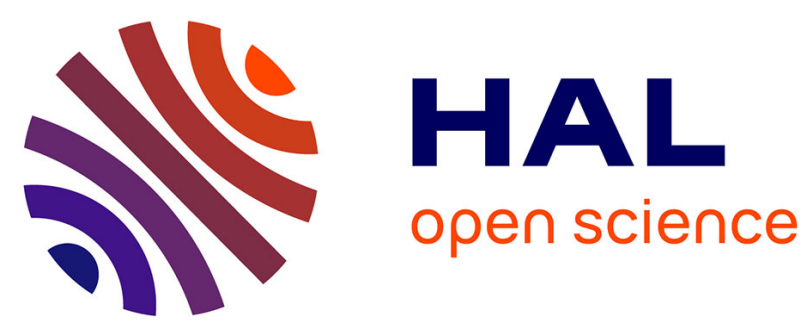

\title{
Towards a Smart Cloud Gate For Smart Devices
}

Guerari Manel, Amirat Abdelkrim, Mourad Chabane Oussalah, Laouar Ridda

\section{To cite this version:}

Guerari Manel, Amirat Abdelkrim, Mourad Chabane Oussalah, Laouar Ridda. Towards a Smart Cloud Gate For Smart Devices. IEEE Innovative systems and Technolgies for the future (CIST'14), Oct 2014, Tetuan-Chefchaouen, Morocco. hal-01067943

\section{HAL Id: hal-01067943 \\ https://hal.science/hal-01067943}

Submitted on 29 Sep 2014

HAL is a multi-disciplinary open access archive for the deposit and dissemination of scientific research documents, whether they are published or not. The documents may come from teaching and research institutions in France or abroad, or from public or private research centers.
L'archive ouverte pluridisciplinaire HAL, est destinée au dépôt et à la diffusion de documents scientifiques de niveau recherche, publiés ou non, émanant des établissements d'enseignement et de recherche français ou étrangers, des laboratoires publics ou privés. 


\title{
Towards a Smart Cloud Gate For Smart Devices
}

\author{
Manel Gherari ${ }^{\mathrm{a}}$, Abdelkrim Amirat ${ }^{\mathrm{b}}$, Mourad Oussalah $^{\mathrm{c}}$ and Ridda Laouar ${ }^{\mathrm{a}}$ \\ ${ }^{a}$ LAMIS Laboratory, university of Tebessa, Algeria \\ ${ }^{\mathrm{b}}$ LIM Laboratory, university of Souk-Ahras, Algeria \\ ${ }^{c}$ LINA Laboratory, university of Nantes, France
}

\begin{abstract}
Mobile Cloud Computing (MCC) is a new paradigm that has been introduced as an optimal solution for mobile technology's issues. Mobile technology aims to exploit the advantages offered by the Cloud in order to provide ubiquitous PC-like functionalities to mobile users. It is noteworthy that despite the benefits associated with the adoption of the Cloud by mobile technology the gate to the Cloud remains frozen. This means that mobile applications often use the same services without having an update of the novelty in Cloud. Thus, applications lack awareness of new services which are more advantageous in terms of features and qualities than the currently used ones. To enable mobile applications to exploit the Cloud intelligently, we propose Smart Mobile Cloud Architecture (SMCA). We consider this new architecture as referential allowing MCC users to have a full awareness of both contexts (Cloud and Mobile) at the same time. We introduce a new concept called Smart Cloud Gate (SCG), which aims to profile both mobile applications and the Cloud to extract knowledge that will be used as a criteria to select the appropriate services, which will be suggested to mobile applications and give each different application the appropriate view of the Cloud.
\end{abstract}

Keywords-Mobile Cloud computing; Cloud computing; Smart Mobile Cloud Architecture; Smart Cloud Gate.

\section{INTRODUCTION}

Mobile devices have become an increasingly important part in human life; they not only facilitate communication that is limited by neither time nor space. Nowadays, mobile applications are becoming more and more ubiquitous and provide ever richer functionalities on mobile devices.

As predicted by Walsh [1] Smart Phones will overtake PCs as the most used means of access to the World Wide Web. Thus, mobile devices will become more important and will be involved in almost every aspect of our daily lives. Also, recent market research predicted that by the end of 2014 mobile Cloud application will delivers annual revenues of 20 billion dollars [2].

To achieve this vision we must face the limitations of mobile devices (low bandwidth, small storage capacity, battery's short life, reliability, etc...). In order to overcome these limitations the Cloud paradigm has been intertwined with mobile technology. This new technology aims to bring the benefits of Cloud to mobile devices to improve their performance (e.g. extend battery life, improve data storage, increase processing powers, etc ...).

Mobile Cloud Computing at its simplest refers to the infrastructure where both the data storage and the data processing happen outside of the mobile device (MCC Forum). Therefore, we can consider that mobile Cloud applications represent the next generation of mobile applications

One important feature of mobile Cloud applications is functional collaboration, like mobile social network applications based on data mining. And then, MCC will serve as not only a link that interconnects information from both Cloud and mobile domains, but also as a knowledge resource to help mobile users in their daily activities [3].

In this context, Apple offers a new tool called ICloud [4] as an application that is easy to configure and use. The latter allows the user to access the multimedia data (e.g. music, photos, documents, etc ...) from any Apple device, data synchronization at all devices (automatic update) and remote access to documents in the Cloud. Although Apple almost fully exploit the Cloud but the features offered by ICloud application remain limited for users of this brand only. As the services library offered by Apple is very limited and non-extensible.

Before proceeding to the development of Cloud applications for Smart Phones, it is essential to consider the challenges posed by the Cloud paradigm as Cloud intercommunication, and resource-intensive processing. These challenges are the main concerns of researchers. Flores and Srirama [5] address issues of interoperability between multiple Cloud (Hybrid Cloud), asynchronous delegation of mobile task and dynamic allocation of Cloud infrastructures. Regarding the challenge of communication and access to Cloud resources, Barbera and al. [6] propose a new Offloading Framework CDroid (Cloud-anDroid).

Using profiling techniques, researches target the issue of mobile applications communication with Cloud, their adaptation, and optimizing battery use.[7], [8, 9]. While taking advantage of profiling techniques to profile the Cloud so as to ensure efficient exploitation of the latter by mobile applications, the evolution of mobile Cloud architectures and self adaptation remain challenges not yet addressed.

By Smart Mobile Cloud Architecture (SMCA) that we propose in this research paper we have as ultimate objectives: 1- makes the Cloud exploitable by any type of mobile device. 
2- Provides to a given mobile application a set of required Cloud services, according to the criteria extracted from the information resulted from profiling the Cloud and the mobile Cloud applications.

To fulfill our objectives we propose two types of middleware. First: Smart Cloud Gate (SCG) as a middleware responsible for filtering the Cloud and offers different Cloud's views to different applications based on the profile of the latter. Second: Smart Mobile Cloud Middleware (SMCM) that is in charge of managing the complexity of the communication between the mobile and hybrids Cloud, by implementing a cloud services composition mechanism.
The rest of this paper is organized as follows: Section 2 discusses the basic concepts building the context of our work. In Section 3 we detail the proposed approach. The state of art of related work will be presented in Section 4 and finally a conclusion on our work will be provided in Section 5 .

\section{BASIC CONCEPTS}

As defined above MCC combines the Cloud and the mobile technology, obviously the architecture proposed for this new paradigm includes the basic concepts of the Cloud and those of mobile [10]. All of these concepts and the general configuration of their interactions are presented in Figure 1.

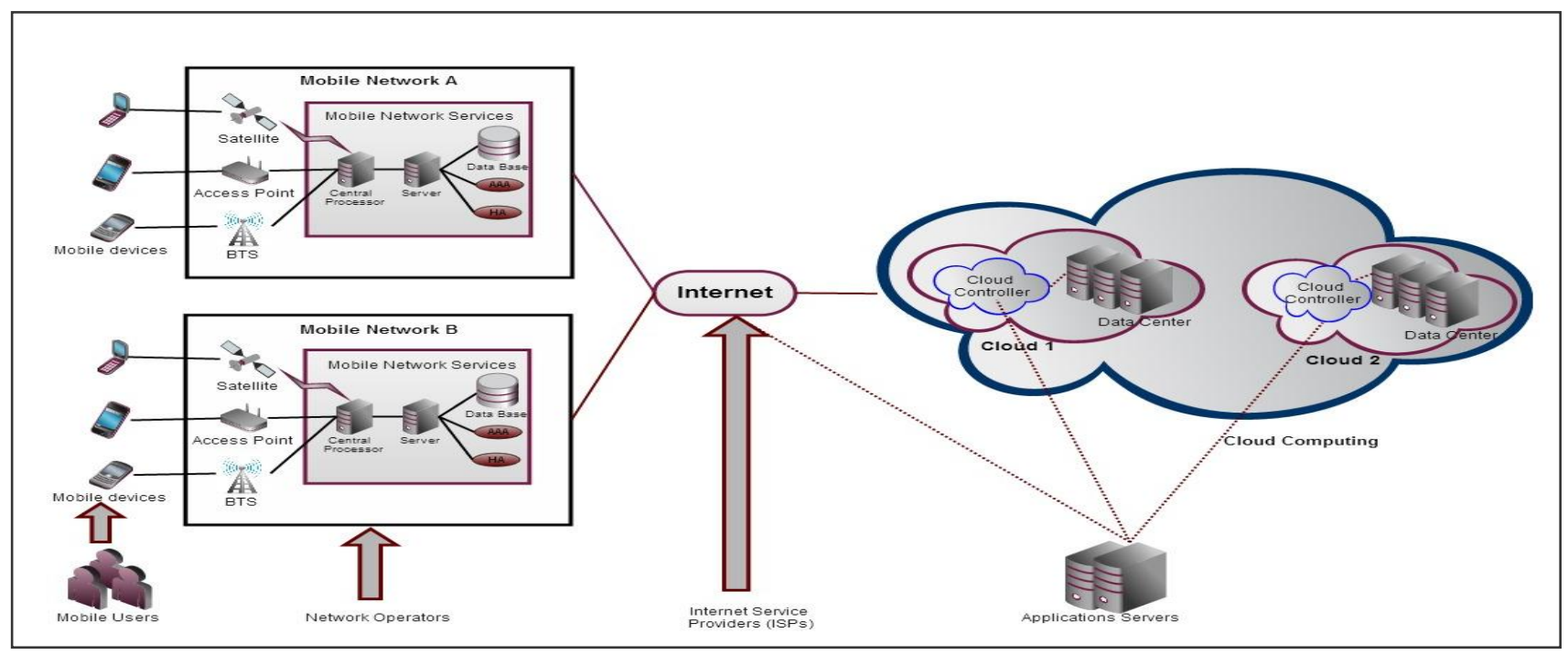

Figure 1- Mobile Cloud Computing Architecture.

Mobile devices are connected to the mobile networks via base stations (e.g., base transceiver station (BTS), access point, or satellite) that institute and control the connections between the networks and mobile devices. Central processors that are connected to servers providing mobile network services, receive mobile user's request and information. Based on the home agent (HA) and data stored in databases, mobile network operators can provide authentication, authorization, and accounting services to mobile users. Finally, the user requests are delivered to a Cloud through the Internet. In the Cloud side, Cloud controllers process the requests and provide mobile users the requested Cloud services.

In the following subsection we will present different access models to the Cloud. Delegation and Offloading and some related works that address challenges posed by these access models.

\section{Access Model to the Cloud}

To improve the performance of mobile devices and address its inherent problems such as resource scarcity, frequent disconnections, and mobility. Cloud was integrated in mobile computing to enable executing mobile applications on resource providers external to the mobile device [11]. This integration is done via the middleware that functions as a mediator between the Cloud and the mobile application. The middleware is in charge of controlling and coordinating the interactions (on/offline) between Cloud infrastructures and mobile devices. We present two types of Cloud access models: Offloading and delegation.

In the delegation model, so the mobile task (MT) could be delegated to the Cloud by directly invoking the Cloud service from the mobile device, the integration of different Cloud services within the mobile application is done in service oriented manner. Therefore, it should be noted that operations delegated to the Cloud generate a significant Overhead and are time-consuming, and computationally unfeasible for offline devices. This integration model requires having always available network connectivity [5].

The general architecture of the delegation model aims to integrate Cloud functionalities that are provided as Web services in Software as a Service (SaaS) level using specialized middleware. 
Basically, in mobile code offloading, a mobile application may be partitioned explicitly for remote execution by a software developer or implicitly by an automated mechanism so that at runtime the mobile components (MC) are identified and analyzed by an offloading decision engine. Deciding whether to offload a mobile component or not is done by a decision engine, basically, this latter measure the mobile device's befits obtained from offloading by profiling multiple local aspects of the device (e.g. bandwidth connectivity, size of data, etc.) and applies certain logic over them (e.g. linear programming) [12].

Code offloading general architecture encourages a design in which, the Cloud provides the virtual computational resources and the mobile introduces the partition strategies, the decision logic based on the mobile local context (e.g. network bandwidth), and the basic implementation primitives that enables to synchronize a mobile application stack with a virtual machine running in the Cloud (e.g. Android x86) [13].

\section{PROPOSED APPROACH}

The Cloud is not only considered as a resource for provisioning services on demand via the Internet but it has its own intrinsic characteristics, such as elasticity that gives an illusion of infinite resources and promotes task parallelism. So a perfect mobile Cloud Framework must take advantage of all these features.

Despite the increasing usage of the Cloud by mobile application, exploiting its full potential is not done in systematic and well structured manner. Furthermore both mobile's and Cloud's contexts are managed separately, therefore the benefits of the Cloud upon mobile technology remains limited. Addressing this challenges to question that must be asked is; how to take advantage of the Cloud in a smart manner? Our suggested Answer is an environment composed of three levels: Architectural level, the intermediate level composed of two middleware Smart Cloud Gate and Smart Mobile Cloud Middleware. Finally the last level consists of mobile level (i.e. application level). An overview of the proposed environment is shown in Figure 2.

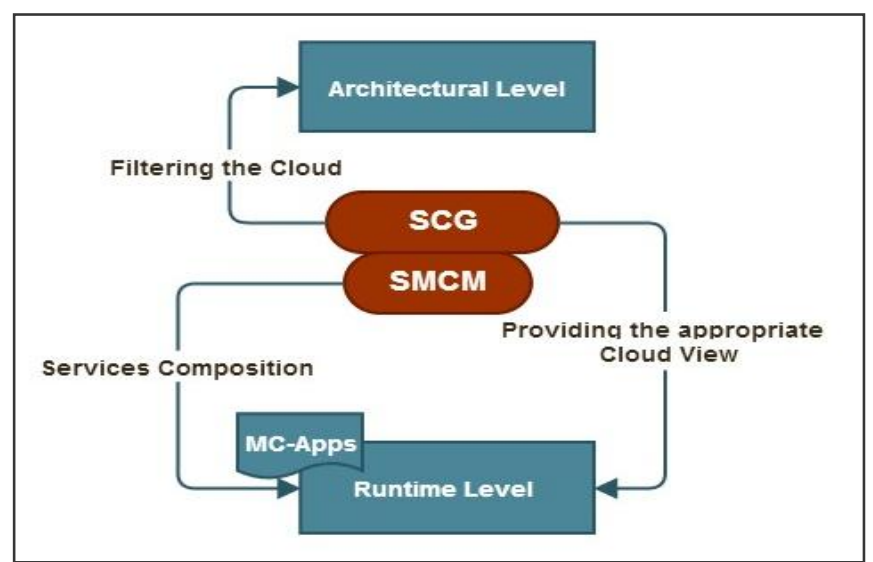

Figure 2- An Overview of Smart Cloud Environment.

In this paper we will discuss the general view of the architecture with a particular focus on Smart Cloud Gate.

Smart Mobile Cloud Architecture (SMCA) is a reference architecture which aims to make the Cloud usable in a systematic and well structured manner by all types of mobile devices. The purpose of having addressed this challenge at the architectural level is; to offer a generic solution and be able to manage the context of Cloud and the context of the mobile simultaneously, while treating a given problem.

The basic concepts constituting our proposed architecture are presented in Figure 3 as a meta-model described by UML class diagram. SMCA covers the main concepts of mobile and Cloud.

- $\quad$ The Cloud Side is composed of different Cloud types (private, community, public, hybrid Cloud),

- $\quad$ Service: provisioned services could be applications or software as services (SaaS), platforms as services

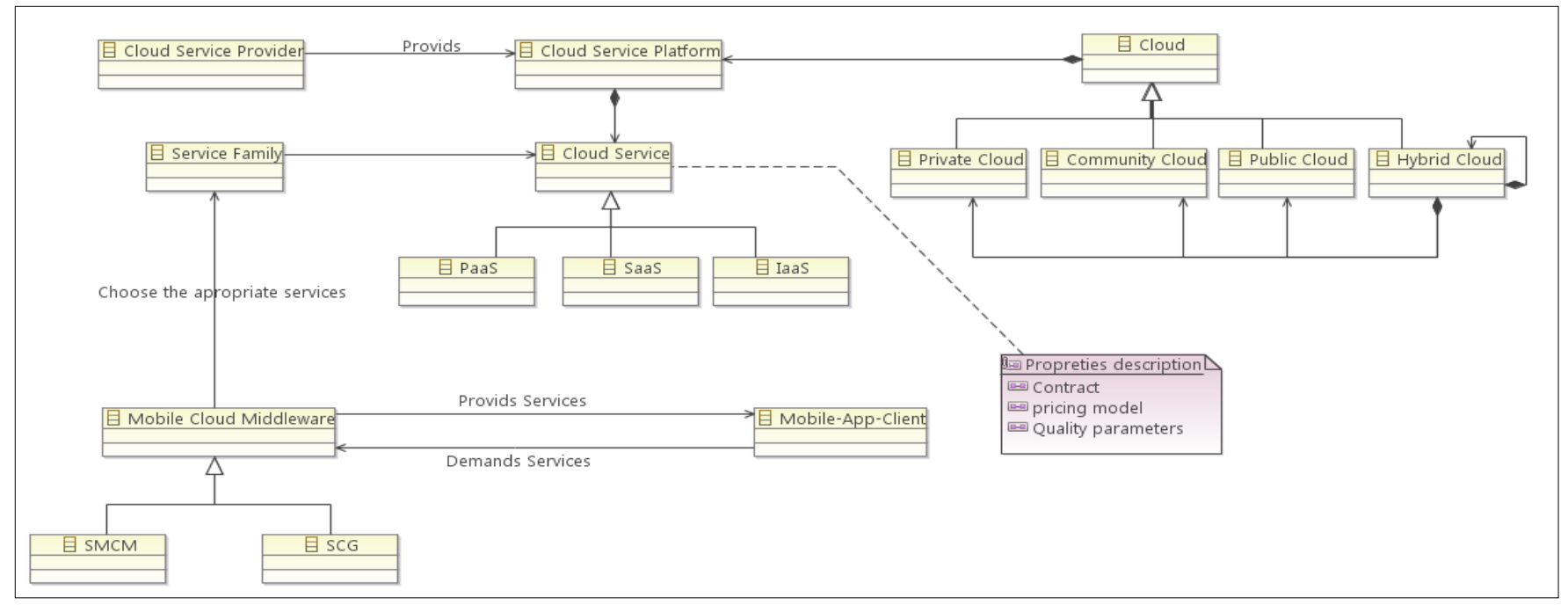

Figure 3- Smart Mobile Cloud Architecture Meta-Model. 
- (PaaS) and infrastructures as services (IaaS). QoS, service contract, and pricing model are represented as annotations,

- Service Provider: an enterprise or an individual provisioning service on demand following as you-useprice metric. Service platform is composed of several services,

- Service Consumer: Is the mobile Cloud application (Mobile-App-Client),

- Mobile device: is an abstract class that encompasses information about Operating System's information (e.g type like android, Windows phone).

- Smart Cloud Gate: is the gate to the Cloud represented as a middleware that customizes services to customers according to their preferences,

- Smart mobile Cloud Middleware(SMCM): mobile apps are no longer satisfied with one service, they uses several different services provided by hybrid clouds, to address communication issues between the mobile and the cloud, SMCM implements a cloud services composition mechanism,

- Service family: is a data warehouse that stores all necessary information regarding Cloud services. This information will serve later as profiling parameters.
SMCA is based on profiling technique to support the functioning of the SCG. Profiling is adopted with the objective of having knowledge about the Cloud and mobile applications, in order to provide to Cloud applications the services they need in the form of a specific Cloud view for each application.

Due to the space constraint in this paper, we do not return to the detailed definitions of these concepts. However, further explanation of Cloud's computing concepts are widely discussed in [14]

The general schema of SMCA functioning is presented in Figure 4. In this schema we define three parts: the Cloud part, the mobile part and the middleware Smart Cloud Gate (SCG) between the first two parts. SCG is a gateway to the Cloud that provides a personal perspective, based on the profiling technique and data mining from a Data warehouse. SCG middleware categorize the profiles of several Clouds and mobile application into a data warehouse using a Cluster. DBServer decides after the data mining from Profile Data Warehouse the set of services that compose a Cloud perspective (Cloud View) proposed for a given mobile application.

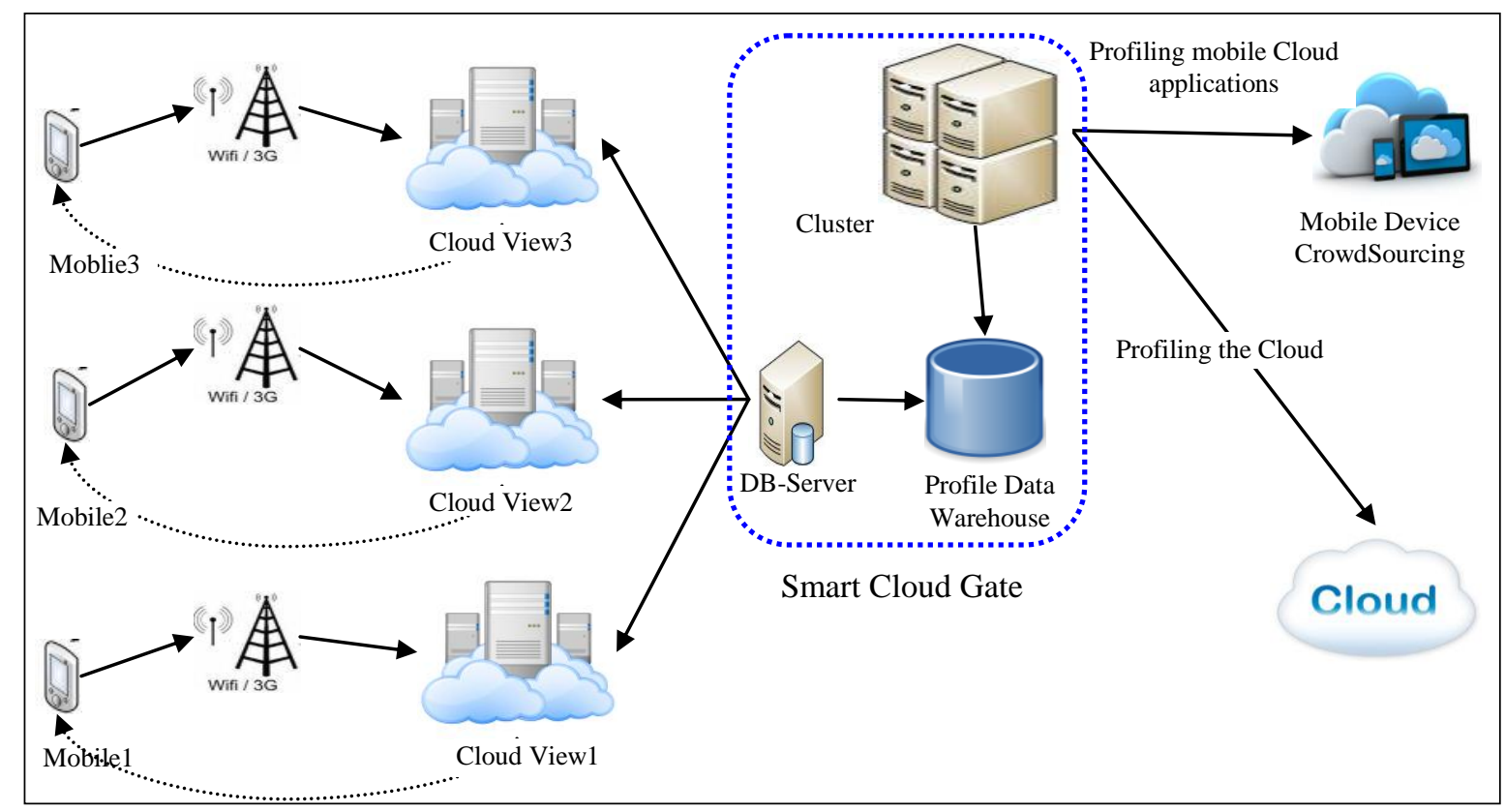

Figure 4- Smart Cloud Gate Architecture.

In our approach the profiling is based on the study of applications' compatibility with the Cloud service platforms, pricing models, qualities of service (QoS) and the history of Cloud's access by mobile devices.
According to SMCA; the client-application accesses the Cloud via Smart Cloud Gate that we consider as a dynamic portal, displaying only the list of services that can be useful for the application, based on the results of profiling made by DBServer. 
To validate our approach, a prototype of SCG as an Eclipse plug-in is under development. We plan to test the proposed virtual Cloud middleware using the Framework Cloudsim developed by the team of R. Buyya in his CloudLab laboratory [15].

\section{RELATED WORKS}

In this section we present a state of art on the related work to our problem by focusing on certain limits that we have tried to compliment by our contributions.

The purpose of Mobile Cloud Computing is to improve the performance of mobile devices by leveraging the Cloud. Among the main concerns of researchers in this area is how to extend battery life? How to manage the complexity of processing and communication in the Cloud? and more other concerns.

Flores and Srirama [5] propose Mobile Cloud Middleware (MCM), which addresses the issues of interoperability between multiple Cloud, transparent delegation and asynchronous execution of mobile tasks that require intensive resource processing and dynamic allocation of Cloud infrastructure. The developed MCM is the intermediary between mobile devices and the Cloud in order to enable asynchronous delegation of mobile task to the Cloud resources. Unlike our vision for Smart Mobile Cloud Middleware in which we aim to make it implements the two access models (the delegation and Offloading), then use the profiling technique and data mining to choose the right model for a connection to the Cloud at a specific time.

In Offloading model, basically when deciding which application's component to offload into the Cloud, the majority of the proposed approaches rely on local aspects as reference to make a decision (e.g. bandwidth, and battery life). While the Cloud context can enhance the decision criteria, and yet not taken in consideration. Hence, Flores and al. respond to this limit by providing Evidence Mobile Cloud Offloading (EMCO), which can be understood as a solution to overcome the problems of adaptive partitioning of applications, decision making concerning the offloading and dynamic resource allocation in the Cloud. [12].

To increase the gain of offloading applications that require intensive calculations; Marco and al. [6] propose a new Framework CDroid (Cloud-anDroid) for Android. CDroid is designed to enable code offloading of intensive communication application like Facebook mobile, twitter and online gaming. In CDroid each mobile device is associated with its Cloud counterpart similar to the real mobile device. This approach gives the illusion of having the Cloud too close to mobile device.

Unlike previously mentioned solutions that address the challenges of communication in Cloud and tend to improve the access models (Offloading / Delegation), Huerta and Lee' vision [16] focuses on communication with mobile virtual Cloud based on mobile devices instead of current Cloud infrastructure, which can be time consuming and very expensive (e.g. price of services required and price of the
connection).The proposed Framework mimics a traditional Cloud provider using mobile devices in proximity to users.

We believe that the first step to use the Cloud, in a methodical and rational manner; is to have knowledge about Cloud clients to provide only the services that responds to their changing needs. Relying on this idea; Garg and al. [17] propose Service Measurement Index Cloud (SMICloud); a Framework for classifying Cloud services according to the following criteria: Accountability, Agility, Assurance of Service, Cost, Performance, Security and Privacy, and Usability. SMICloud is a Framework that helps Cloud clients to find the most appropriate Cloud provider.

The work presented in this section address issues of one context at a time (mobile context or Cloud context). Although the proposed middleware by Flores and Srirama [5] is considered as a mediator between the Cloud and the mobile device, but it only focus on issues related to mobile context: enhance the delegation benefits, hiding the complexity while dealing with several services providers (hybrid Cloud).

Only been inspired from the Cloud in order to have a virtual one composed of mobile devices, Huerta and Lee [16] have missed the fact that even addressing the time and cost problems of connection with Cloud, limited mobile resources are unrivaled with Cloud intensive resources. In this context we consider that the proposed solution by the latter, as a plan $\mathrm{B}$ in case of an emergency that requires the use of external resources for a given treatment.

\section{CONCLUSION}

The synergy of the paradigm Cloud and mobile technology results an improvement of mobile devices' performances. Unfortunately, most researches focus on one context at a time (Cloud or Mobile), which generates a low exploitation of the Cloud's benefits and an ill utilization of intensive resources available that it provides. Motivated by this limit we have proposed in this proceeding research a reference architecture, Smart Mobile Cloud Architecture that offers a generic solution which enable managing both Cloud and mobile contexts simultaneously, also taking advantage of the Cloud in a smart way using Smart Cloud Gate middleware that customize the Cloud by taking as a reference the users requirements in order to offer to each user the appropriate perspective on the Cloud.

The environment proposed in this work is composed of three levels; Architectural level, the intermediate level; composed of two middleware Smart Cloud Gate and Smart Mobile Cloud Middleware, finally the last level consist of mobile level. In this paper we discussed the general architecture with a particular focus on Smart Cloud Gate.

In our future work we plan to address the second component of layer 2 (SMCM) which will be responsible for communication between the mobile and hybrid clouds, implementing a composition mechanism.

\section{REFERENCE}

1. Walsh, M., Gartner: Mobile to outpace desktop web by 2013. Online Media Daily, 2010.

2. Beccue, M. and D. Shey, Mobile Cloud Computing. ABI Research, 2009. 
3. Huang, D., Mobile cloud computing. IEEE COMSOC Multimedia Communications Technical Committee (MMTC) E-Letter, 2011. 6(10): p. 27-31.

4. Apple. ICloud. 2014.

5. Flores, H. and S.N. Srirama, Mobile cloud middleware. Journal of Systems and Software, 2013.

6. Barbera, M.V., et al., Mobile Offloading in the Wild: Findings and Lessons Learned Through a Real-life Experiment with a New Cloudaware System. Proc. of IEEE INFOCOM 2014, 2014.

7. Qian, F., et al. Profiling resource usage for mobile applications: a crosslayer approach. in Proceedings of the 9th international conference on Mobile systems, applications, and services. 2011. ACM.

8. Flinn, J. and M. Satyanarayanan. Powerscope: A tool for profiling the energy usage of mobile applications. in Mobile Computing Systems and Applications, 1999. Proceedings. WMCSA'99. Second IEEE Workshop on. 1999. IEEE.

9. Narayanan, D., J. Flinn, and M. Satyanarayanan. Using history to improve mobile application adaptation. in Mobile Computing Systems and Applications, 2000 Third IEEE Workshop on. 2000. IEEE.

10. Dinh, H.T., et al., A survey of mobile cloud computing: architecture, applications, and approaches. Wireless communications and mobile computing, 2011
11. Fernando, N., S.W. Loke, and W. Rahayu, Mobile cloud computing: A survey. Future Generation Computer Systems, 2013. 29(1): p. 84-106.

12. Flores Macario, H.R. and S. Srirama. Adaptive code offloading for mobile cloud applications: Exploiting fuzzy sets and evidence-based learning. in Proceeding of the fourth ACM workshop on Mobile cloud computing and services. 2013. ACM.

13. Flores, H., S.N. Srirama, and R. Buyya, Computational Offloading or Data Binding? Bridging the Cloud Infrastructure to the Proximity of the Mobile User. 2014.

14. Mell, P. and T. Grance, The NIST definition of cloud computing (draft). NIST special publication, 2011. 800(145): p. 7.

15. Calheiros, R.N., et al., CloudSim: a toolkit for modeling and simulation of cloud computing environments and evaluation of resource provisioning algorithms. Software: Practice and Experience, 2011. 41(1): p. 23-50.

16. Huerta-Canepa, G. and D. Lee. A virtual cloud computing provider for mobile devices. in Proceedings of the 1st ACM Workshop on Mobile Cloud Computing \& Services: Social Networks and Beyond. 2010. ACM.

17. Garg, S.K., S. Versteeg, and R. Buyya, A framework for ranking of cloud computing services. Future Generation Computer Systems, 2013. 29(4): p.1012-1023. 